\title{
CONSTRUCTION OF WEAK SOLUTIONS OF THE NAVIER-STOKES EQUATION IN A NONCYLINDRICAL DOMAIN ${ }^{1}$
}

\author{
BY HIROSHI FUJITA AND NIKO SAUER
}

Communicated by Jürgen K. Moser, October 4, 1968

1. Introduction and the result. Consideration of the motion of a viscous fluid in a vessel with moving walls or in a vessel containing rigid bodies moving through the fluid leads us to the initial value problem for the Navier-Stokes equation in a noncylindrical domain in $(t, x)$-space. This problem will be denoted by ( $\operatorname{Pr} . \mathrm{NC})$. Let $\Omega(t)$ $\subset R^{m}$ ( $m=2$ or 3 ) be the domain filled by the fluid at time $t$ and let $\Gamma(t)$ be the boundary of $\Omega(t)$. We shall be concerned with the flow for the time interval $[0, T](T>0)$. We put

$$
\hat{\Omega}=\bigcup_{t \in[0, T]} \Omega(t) \quad \text { and } \quad \hat{\Gamma}=\bigcup_{t \in[0, T]} \Gamma(t)
$$

(Pr. NC) in its classical form is to find out the velocity field $u=u(t, x)$ and the pressure $p=p(t, x)$ which satisfy the following.

$$
\begin{aligned}
u_{t} & =\Delta u-\nabla p-(u \cdot \nabla) u+f(t, x) & & \text { in } \hat{\Omega}, \\
\nabla \cdot u & \equiv \operatorname{div} u=0 & & \text { in } \hat{\Omega}, \\
u & =\beta(t, x) & & \text { on } \hat{\Gamma}, \\
u & =a(x) & & \text { in } \Omega(0) .
\end{aligned}
$$

$f, \beta$ and $a$ are given (vector) functions. Here and hereafter the differential operators $\Delta$ and $\nabla$ mean those for $x$ variables only. The special case of (Pr. NC) with $\Omega(t)$ independent of $t$ will be denoted by (Pr. C). The objective of the present note is to extend $E$. Hopf's existence theorem (cf. [1]) for weak solutions from (Pr. C) to (Pr. NC). Intending to emphasize rather the straightforwardness of the method than the generality of the result, we here make the simplifying assumptions (A1)-(A3). These assumptions will be released completely or weakened considerably in a forthcoming paper where we shall give full details of our study.

1 This research was partly supported by the National Science Foundation, Grant NSF-GP-8114 while the authors were visiting members at the Courant Institute of Mathematical Sciences. The first author was subsequently supported as a visiting professor in the Mathematics Research Center, United States Army, Madison, Wisconsin, under Contract No. DA-31-124-ARO-D-462. 
(A1). $f \equiv 0$ and $\beta \equiv 0$. (The latter may not be physically reasonable!)

(A2). For some fixed bounded domain $B \subset R^{m}$ we have $(\bar{\Omega}(t)) \subset B$.

(A3). $\Gamma(t)$ is composed of a finite number of smooth simple closed surfaces (curves if $m=2$ ). Also $\Gamma(t)$ changes smoothly as $t$ increases. (Unfortunately we do not have enough room in this short paper to specify the "smoothness" involved in (A3). We only note that (A3) is needed mainly for the proof of Lemma 5.)

We now state our main theorem, although the definition of weak solutions and that of $H_{0}(\Omega(0))$ are to be given in $\$ 3$.

Theorem 1. Assume (A1)-(A3). If $a \in H_{\sigma}(\Omega(0))$, then there is a weak solution $u$ of $(\operatorname{Pr} . N C)$.

Remark 2. If $m=2$, the uniqueness theorem by J. O. Sather and $\mathrm{J}$. Serrin (cf. [2]) is applicable to our weak solutions.

The authors wish to thank Professor Masatake Kuranishi for valuable advice in connection with the proof of Lemma 5 which is, however, too long to be contained in this paper.

2. Approximating problems. The basic idea of our method is to approximate ( $\operatorname{Pr} . \mathrm{NC}$ ) by the following initial value problem $(\operatorname{Pr} . \mathrm{AP})_{n}$ in the cylindrical region $\hat{B}=[0, T] \times B, n$ being an arbitrary positive integer;

$$
\begin{aligned}
u_{t}^{n} & =\Delta u^{n}-\nabla p^{n}-\left(u^{n} \cdot \nabla\right) u^{n}-n \chi(t, x) u^{n} & & \text { in } \hat{B}, \\
\nabla \cdot u^{n} & =0 & & \text { in } \hat{B}, \\
\left.u^{n}\right|_{\partial B} & =0 & & \text { on }[0, T] \times \partial B, \\
\left.u^{n}\right|_{t=0} & =\bar{a}(x) & & \text { in } B .
\end{aligned}
$$

Here $\chi(t, x)$ is the characteristic function of $E=\hat{B}-\hat{\Omega}$ and $\bar{a}$ means the natural extension of $a$ to $B$, that is, $\bar{a}=a$ in $\Omega(0)$ and $\bar{a}=0$ in $B-\Omega(0)$. We note that if $\left\{u^{n}\right\}$ or a subsequence of it converges to $\bar{u}$ as $n \rightarrow \infty$ in some sense or other, then the limit $\bar{u}$ is a good candidate of the solution of (Pr. NC). In fact, it is easy to derive the following a priori estimate for $u^{n}$;

$$
\left\|u^{n}(t)\right\|^{2}+2 \int_{0}^{t}\|\nabla u\|^{2} d s+2 n \int_{0}^{t}\left(\chi u^{n}, u^{n}\right) d s \leqq\|\bar{a}\|^{2},
$$

$t$ being any number in $[0, T]$. Here \|\| denotes the $L_{2}(B)$-norm of vector functions and tensor functions. Other symbols in (1) are understood. From (1) it is obvious that $u^{n} \rightarrow 0$ strongly in $L_{2}(E)$ as $n \rightarrow \infty$. Thus the restriction of the limit $\bar{u}$ onto $\hat{\Omega}$ will give the required 
solution. Our actual proof of Theorem 1 is to justify this argument.

3. Definition of weak solutions. First of all we introduce some symbols. In this section $u$ and $\phi$ stand for vector functions. Let $\Omega$ be any bounded domain in $R^{m}$. Then

$$
D_{\sigma}(\Omega)=\left\{\phi \in C^{\infty}(\Omega) \mid \operatorname{supp} \phi \subset \Omega \text { and } \nabla \cdot \phi=0\right\} .
$$

$H_{\sigma}(\Omega)=$ the completion of $D_{\sigma}(\Omega)$ under the $L_{2}(\Omega)$-norm. $H_{\sigma}^{1}(\Omega)=$ the completion of $D_{\sigma}(\Omega)$ under the $W_{2}^{1}(\Omega)$-norm or equivalently, under the Dirichlet norm. The inner product in $L_{2}(\Omega)$ is written as $(,)_{\Omega}$ or simply as $($,$) when \Omega$ is understood.

Now let $G$ be either $\hat{B}$ or $\hat{\Omega}$. Note that $G$ is closed at the 'top' and at the 'bottom.' We put

$$
\hat{D}_{\sigma}(G)=\left\{\phi \in C^{\infty}(G) \mid \operatorname{supp} \phi \subset G, \nabla \cdot \phi=0\right\},
$$

$\hat{H}_{\sigma}^{1}(G)=$ the completion of $\hat{D}_{\sigma}(G)$ under the norm

$$
\left(\iint_{G}|\nabla \phi(t, x)|^{2} d t d x\right)^{1 / 2} \text {. }
$$

Definition 3. $u=u(t, x)$ defined in $\hat{\Omega}$ is called a weak solution of (Pr. NC) if (i)-(iii) hold.

(i) $u \in \hat{H}_{\sigma}^{1}(\hat{\Omega})$.

(ii) For every $t \in[0, T], u(t) \equiv u(t, \cdot) \in H_{\sigma}(\Omega(t))$ and $\|u(t)\|$ is bounded on $[0, T]$.

(iii) For every $\phi \in \hat{D}_{\sigma}(\Omega)$ vanishing at $t=T$, the equality

(2) $F(u, \phi) \equiv \int_{0}^{T}\left\{\left(u, \phi_{t}\right)+(u, \Delta \phi)+(u,(u \cdot \nabla) \phi)\right\} d t=-(a, \phi(0))$ is satisfied, where $\phi(0)=\phi(0, \cdot)$.

Definition 4. $u^{n}=u^{n}(t, x)$ defined in $\hat{B}$ is called a weak solution of $(\operatorname{Pr} . \mathrm{AP})_{n}$ if (i)-(iii) hold.

(i) $u^{n} \in \hat{H}_{\sigma}^{1}(\hat{B})$.

(ii) For every $t \in[0, T], u^{n}(t) \equiv u^{n}(t, \cdot) \in H_{\sigma}(B)$ and $\left\|u^{n}(t)\right\|$ is bounded on $[0, T]$.

(iii) For every $\phi \in \hat{D}_{\sigma}(\hat{B})$ vanishing at $t=T$, the equality

$$
F\left(u^{n}, \phi\right)=n \int_{0}^{T}\left(\chi u^{n}, \phi\right) d t-(\bar{a}, \phi(0))
$$

is satisfied.

4. Outline of the proof of Theorem 1. The basic idea of the proof was mentioned in $\$ 2$. We briefly indicate necessary steps in the proof. 
1st Step. Exactly as in [1], we prove that $(\operatorname{Pr} . \mathrm{AP})_{n}$ has a weak solution $u^{n}$ for which the inequality (1) holds.

$2 n d$ Step. Following the argument in [1] with a slight modification we show that it is possible to select a subsequence $\left\{v^{n}\right\}$ of $\left\{u^{n}\right\}$ such that $v^{n}$ converges to $\bar{u}$ weakly in $\hat{H}_{\sigma}^{1}(\hat{B}), v^{n} \mid E$ converges to 0 strongly in $L_{2}(E)$, and that for every compact set $K \subset \hat{\Omega}$ the restriction of $v^{n}$ to $K$ converges strongly in $L_{2}(K)$. It is easy to verify that $u=\vec{u} \mid \hat{\Omega}$ satisfies (2).

3rd Step. Use the following lemma to show that $u \in \hat{H}_{\sigma}^{1}(\hat{\Omega})$. We recall (A3).

LemmA 5. Let $w \in \hat{H}_{\sigma}^{1}(\hat{B})$. If $w=0$ in $E=\hat{B}-\hat{\Omega}$, then $w \mid \hat{\Omega} \in \hat{H}_{\sigma}^{1}(\hat{\Omega})$.

4 th Step. Following the argument in [2] or reexamining the procedure in the 2 nd step, we realize that $u(t)$ satisfies the second condition of Definition 3 after possible redefinition on a null set of $t$.

\section{REFERENCES}

1. E. Hopf, Über die Anfangswertaufgabe für die hydrodynamischen Grundgleichungen, Math. Nachr. 4 (1951), 213-231.

2. J. Serrin, Initial value problem for Navier-Stokes equations, nonlinear problems, Univ. of Wisconsin Press, Madison, 1963, pp. 69-98.

The University of Tokyo, Japan and

National Research Institute for Mathematical Sciences, Pretoria, SOUTH AFrICA

\section{ERRATUM, VOLUME 74}

C. A. Berger and L. A. Coburn, $C^{*}$-algebras of translations and multipliers, pp. 1008-1012.

The proof of Lemma 2 is incorrect. Theorem 1 remains correct provided we add the hypothesis that $G$ has an element which acts ergodically by translation. In this case, we can apply the pointwise ergodic theorem and the Lebesgue dominated convergence theorem in place of Lemma 2. 